\title{
Patient specific respiratory motion model using two static CT images
}

\author{
Tengfei Wang \\ Anhui Province Key Laboratory of \\ Medical Physics and Technology, \\ Institute of Health and Medical \\ Technology, \\ Hefei Institutes of Physical Science, \\ Chinese Academy of Sciences, \\ Hefei,230031, P. R. \\ wangtengf123@163.com
}

\section{Chen Qi}

Hefei Cancer Hospital, Chinese Academy of Sciences, Hefei, 230031, P. R. China

820119141@qq.com

\author{
Guoren Xia \\ Hefei Cancer Hospital, Chinese \\ Academy of Sciences, Hefei, 230031, \\ P. R. China \\ 534501363@qq.com
}

\author{
Hai $\mathrm{Li}^{\dagger}$ \\ Anhui Province Key Laboratory of \\ Medical Physics and Technology, \\ Institute of Health and Medical \\ Technology, \\ Hefei Institutes of Physical Science, \\ Chinese Academy of Sciences, \\ Hefei,230031, P. R. \\ hli@cmpt.ac.cn
}

\author{
Hongzhi Wang \\ Hefei Cancer Hospital, Chinese \\ Academy of Sciences, Hefei, 230031, \\ P. R. China \\ wanghz@hfcas.ac.cn
}

\begin{abstract}
Computed Tomography (CT) has been extensively used for guiding percutaneous lung biopsy during image-guided intervention. However, due to respiratory motion, there having a difference between static images and current lung. Current studies are using the global model to predict lung movement in real time. This model extracts common features of lung motion based on group-level of imaging data, which overlook the information comes from the randomness of lung movements and individual differences on the features of lung motion and consequently, limit the precision of the model. In order to resolve this issue, patient specific model is proposed and can effectively acquire individual features of lung movement but required $4 \mathrm{D}$ CT image that increases the risk of radiation damage. This paper developed a new patient specific respiratory motion model to establish the mathematical relationship between lung internal motion and external chest surface motion. Only two static 3D CT are needed for a specific patient, and there is no need to collect $4 \mathrm{D}$ CT images. The proposed method combining the advantages of global and patient specific model

Permission to make digital or hard copies of all or part of this work for personal or classroom use is granted without fee provided that copies are not made or distributed for profit or commercial advantage and that copies bear this notice and the full citation on the first page. Copyrights for components of this work owned by others than ACM must be honored. Abstracting with credit is permitted. To copy otherwise, or republish, to post on servers or to redistribute to lists, requires prior specific permission and/or a fee. Request permissions from Permissions@acm.org.

ISAIMS 2021, October 29-31, 2021, Beijing, China

(C) 2021 Copyright is held by the owner/author(s).

ACM ISBN 978-1-4503-9558-8/21/10.

https://doi.org/10.1145/3500931.3501014
\end{abstract}

will ensure better prediction of lung motions and lower the risk of radiation damage. The qualitative and quantitative result show that the proposed model achieved a better balance between predictive accuracy and radiation doses.

\section{CCS CONCEPTS}

-Modeling and simulation • Machine learning

\section{KEYWORDS}

Respiratory motion model, Ridge regression, Artificially 4D CT image

\section{Introduction}

The major problem during the percutaneous lung biopsy [1] procedure is the organ motion caused by respiration [2], which can cause geometrical distortion. To overcome the geometrical distortion, radiologists normally scan multiple CT images on the same position, which increases the radiation dosage. Thus, accurate motion prediction of pulmonary nodules is crucial for image-guided biopsy. Respiratory motion model [3] describes the relationship between the external signals and the lung motion. This model can be used to estimate the lung nodule motion from the surrogate signals during percutaneous lung biopsy procedure.

Recent work for respiratory motion model can be divided into two categories. The first group is global respiratory motion 
model which extracts the entire population common features of lung movement and surrogate signals $[4,5,6,7,8]$. However, due to the difference and randomness of individual lung movement, the characteristics of patient's real movement may be ignored. The second group is patient specific respiratory motion model which uses individual patient's $4 \mathrm{D} \mathrm{CT}$ data to predict lung images [9,10,11]. Although these models can obtain better results, a potential drawback is that the radiation doses of $4 \mathrm{D} C \mathrm{CT}$ are 3-8 times that of normal 3D CT $[12,13]$ and can be harmful to patients. In addition, most of them are based on 3D discrete signals $[14,15,16]$.

To achieve a balance between predictive accuracy and radiation dose, this study developed a new patient-specific respiratory motion model, relying only on two $\mathrm{CT}$ images of inhale and exhale phases. This respiratory model is created using $4 \mathrm{D}$ CT of five patients. Figure 1 is the flowchart of the proposed method. Firstly, the relationship between the deformation field of the individual specific phase (exhalation-inspiration) and the other four patients' corresponding deformation fields is established. Using obtained relationship can create an artificial 4D CT image and reducing radiation damage to patients. Then, a relation between the internal deformation filed motion and the high-dimensional skin surface motion is established. Finally, the difference of deformation field between the predict images and the real images is used to evaluation the motion model.
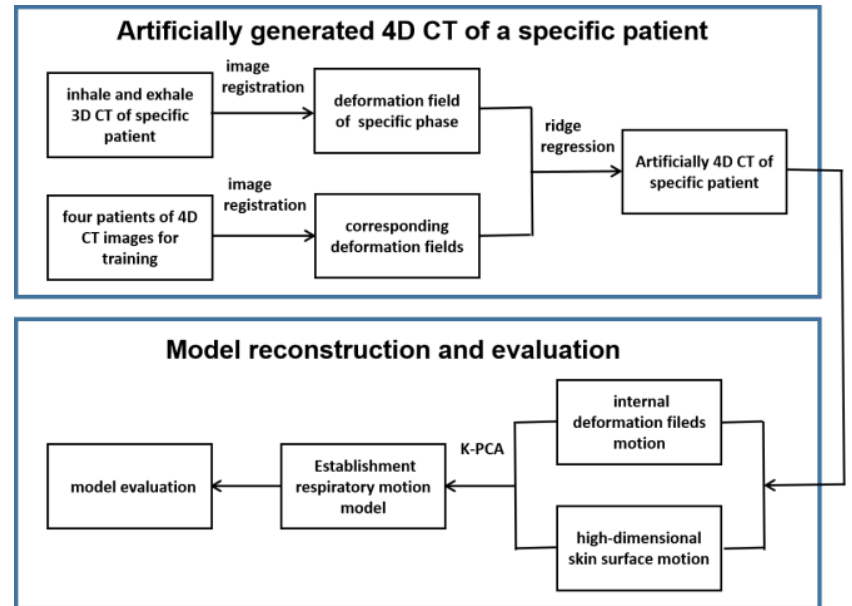

Figure 1: The flowchart of the proposed patient specific respiratory motion model.

\section{MATERIALS AND METHODS}

\subsection{Patient data}

In this study, the clinical data were acquired on a publically database (http://www.dir-lab.com) which provides ten 4D CT image with annotated landmarks. Five patients' 4D CT (case6case10) are chosen for our research. Each patient consists of ten different sequences with dimensions $0.98 \times 0.98 \times 2 \mathrm{~mm}^{3}$ and typical size $512 \times 512 \times 128$ voxels.

\subsection{Artificially generated specific 4D CT images}

In experiments, we used five 4D CT images to generate specific $4 \mathrm{D}$ CT images. One image is chosen as specific patient which only use two 3D CT images (inhale and exhale phase) and the others are training sets. The exhale image was used as the "reference template" due to breathing movement was relatively flat. Using image registration algorithm [17], specific patient's deformation field $\mu_{\mathrm{s}, 1}$ that deforms exhale image into inhale image is generated. With ridge regression algorithm, this deformation field is composed of the other four patients' corresponding deformation fields.

$$
\mu_{\mathrm{s}, 1}=\omega_{1} \mu_{\mathrm{i}, 1}+\omega_{2} \mu_{\mathrm{j}, 1}+\omega_{3} \mu_{\mathrm{z}, 1}+\omega_{4} \mu_{\mathrm{k}, 1}
$$

where $\mu_{\mathrm{i}, 1}, \mu_{\mathrm{j}, 1}, \mu_{\mathrm{z}, 1}, \mu_{\mathrm{k}, 1}$ are the other four patients' deformation fields in exhale image and inhale image. Using obtained four coefficients $\left(\omega_{1}, \omega_{2}, \omega_{3}, \omega_{4}\right)$, we can create ten deformation matrices which describe the deformation corresponding to the specific patient.

$$
\mu_{\mathrm{s}, \mathrm{p}}=\omega_{1} \mu_{\mathrm{i}, \mathrm{p}}+\omega_{2} \mu_{\mathrm{j}, \mathrm{p}}+\omega_{3} \mu_{\mathrm{z}, \mathrm{p}}+\omega_{4} \mu_{\mathrm{k}, \mathrm{p}}
$$

where $p=1 \ldots 10$ is the intermediate respiratory phase displacement field. Then, artificial 4D CT is calculated based on exhale image and ten intermediate displacement field. In addition, other four specific patient's artificial 4D CT is generated by the same way.

\subsection{External skin surface motion}

The 4D CT which obtained from section 2.2 were segmented and extracted skin surface [18]. In addition, an euclidean distance matrix between CT coordinate origin and skin was calculated.This matrix is corresponds to the time of flight camera data [19].

Regions of the skin were chosen on the coronal plane which 10 pixels [Figure 3(a)] were chosen to describe external motion. In addition, the publically DIR database provides internal landmarks correspond to 4D CT image. Each image has 75 landmarks to describe internal motion:

$$
\mathrm{IM}=\frac{1}{\mathrm{n}} \sum_{k=1}^{n} \sqrt{\left(q_{x}^{k}-r_{x}^{k}\right)^{2}+\left(q_{y}^{k}-r_{y}^{k}\right)^{2}+\left(q_{z}^{k}-r_{z}^{k}\right)^{2}}, \quad \mathrm{n}=75
$$

where $q_{x}^{k}, q_{y}^{k}, q_{z}^{k}$ are the kth landmarks' three-dimensional coordinate in template $\mathrm{CT}$, and $r_{x}^{k}, r_{\mathrm{y}}^{k}, r_{\mathrm{z}}^{k}$ are the kth landmarks' three-dimensional coordinate in other phase of CT.

The correlation coefficient (CC) describes a relation between the internal motion and the external skin surface motion. The $\mathrm{CC}$ is defined as following:

$$
\mathrm{CC}=\frac{\left(i-m_{i}\right)\left(e-m_{e}\right)}{\mathrm{s}_{i} \mathrm{~S}_{e}}
$$


where $\mathrm{i}$ is internal motion, e is external motion. $m_{i}$ and $m_{e}$ represent the mean value, $\mathrm{S}_{i}$ and $\mathrm{S}_{e}$ are the standard deviations. These regions which best correlate with the internal motion were used to establish respiratory motion model and the result is presented in section 3.1.

\subsection{Respiratory motion model reconstruction}

From the artificially 4D CT, we obtained the deformation field $\mu$ and the skin surface motion $v$ and define as follows:

$$
\begin{aligned}
& \mu=\left[\mu_{1}, \mu_{2}, \ldots \mu_{m}\right] \\
& \mu_{\mathrm{m}}=\left[\mu_{1,1, \mathrm{~m}}, \mu_{1,2, \mathrm{~m}}, \mu_{1,3, \mathrm{~m}}, \ldots, \mu_{S, 1, m}\right]^{T} \\
& v=\left[v_{1}, v_{2}, \ldots, v_{m}\right] \\
& v_{\mathrm{m}}=\left[v_{1, m}, v_{2, m}, \ldots, v_{N, m}\right]^{T}
\end{aligned}
$$

where $\mu_{\mathrm{s}, \mathrm{i}, \mathrm{m}}$ represents ith (i=1-3) displacement on different coordinate of voxel $s$ at different respiratory phase $m(m=1-10)$,

$\mathrm{S}$ is sum number of voxel, and $v_{\mathrm{n}, \mathrm{m}}$ is nth surrogates' displacement at different respiratory phase $\mathrm{m}, \mathrm{N}=100$.

In order to eliminate the differences between the data, the mean value is subtracted from each respiratory phase. According to the kernel-principle component analysis (K-PCA) model, those eigenvectors $E=\left[e_{1}, e_{2}, \ldots, e_{k}\right]$ with the largest $K$ eigenvalues $\mathrm{W}=\left[\omega_{1}, \omega_{2}, \ldots, \omega_{\mathrm{k}}\right]$ are the principal components used.

$$
\left[\begin{array}{l}
\mu \\
v
\end{array}\right]-\left[\begin{array}{l}
\bar{\mu} \\
\bar{v}
\end{array}\right]=\sum_{\mathrm{k}=1}^{K} \omega_{k}(t) e_{k}=\mathrm{EW}=\left[\begin{array}{c}
\mathrm{E}_{\mu} \\
\mathrm{E}_{v}
\end{array}\right] \mathrm{W}
$$

where $\mathrm{E}_{\mu}$ and $\mathrm{E}_{\nu}$ are the first $3 \mathrm{~S}$ rows and the last $\mathrm{N}$ rows of matrix E, respectively. Mean vectors of deformation field and skin surface motion are denoted as $\bar{\mu}$ and $\bar{v}$. In Eq.(7), W can be eliminated:

$$
\mu(t)-\bar{\mu}=E_{\mu} E_{v}^{-1}(v(t)-\bar{v})=B(v(t)-\bar{v})
$$

where $\mathrm{B}$ is the matrix relation external skin surface motion to internal deformation field.

\subsection{Respiratory motion model evaluation}

In the estimation stage, real $4 \mathrm{D} \mathrm{CT}$ and the correspond skin surface motion are obtained. The error of the respiratory motion model is calculated as:

$$
\mathrm{ME}=\frac{1}{|\Omega|} \sum_{\mathrm{x} \in \Omega}\left|\mu(t)_{\text {real }}-\mu(t)_{\text {predict }}\right|
$$

where $\mu(t)_{\text {real }}$ and $\mu(t)_{\text {predict }}$ are the real deformation field and the deformation field predicted by the model, respectively. $\Omega$ is the voxel domain of the deformation field. $|\Omega|$ is total number.

\section{Results}

\subsection{Evaluation of external skin surface motion correlation}
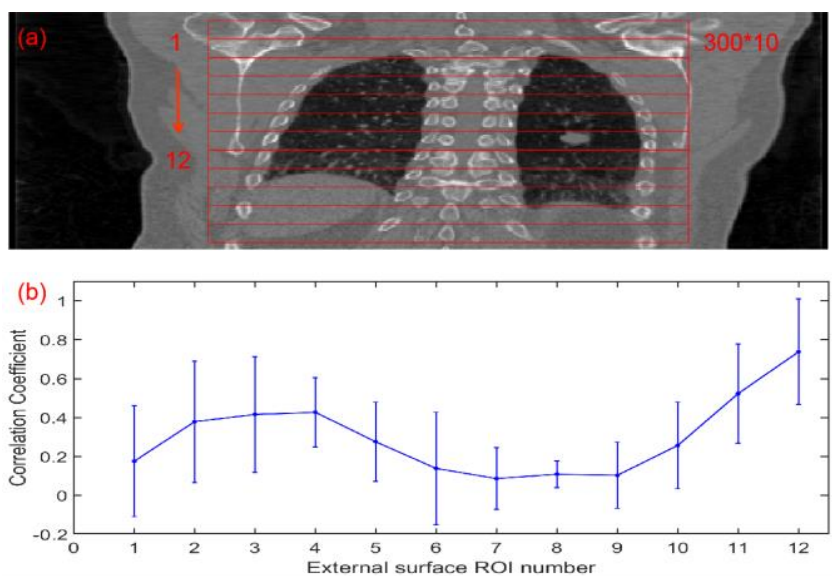

Figure 2: (a) Twelve horizontal rectangular regions placed on the external surface. (b) The mean correlation coefficients for each patients between external areas and all 75 internal landmarks.
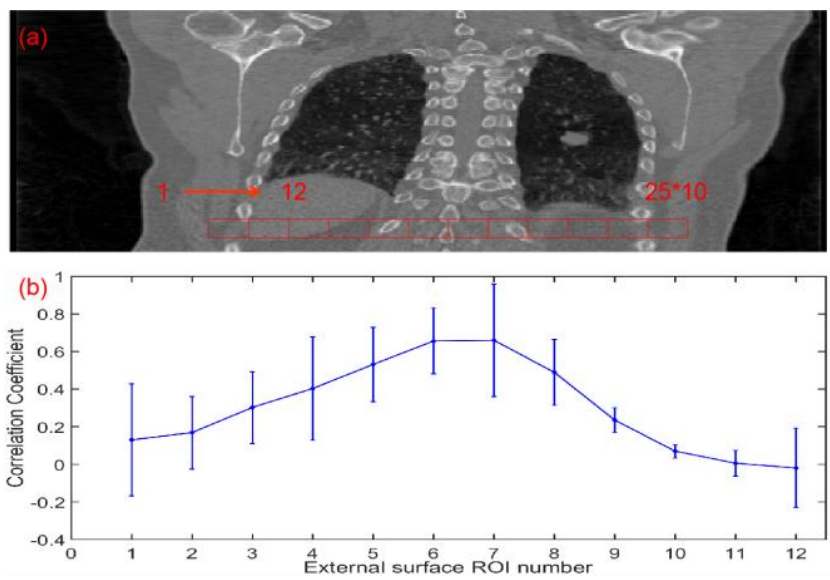

Figure 3: (a) Twelve square regions placed on the external surface. (b) The mean correlation coefficients for each patients between external areas and all 75 internal landmarks. 
Figure 2(a) shows the 12 horizontal rectangular regions selected on the external skin surface. In Figure 2(b), the correlation coefficient between these chosen regions and internal landmarks is shown. The correlation and amplitude of motion in the abdominal region [regions 11 and 12] were found to be higher than other regions. In a further step, Figure 3(a) shows the 12 square regions placed on the abdominal region. Figure 3(b) shows that the central region surrounding the umbilicus [regions 3-9] have higher correlation. From these regions which have higher correlate with the internal landmark, we extracted 100 skin points and are used as external respiratory signals in section 2.4 .

\subsection{Evaluation of the proposed respiratory motion model}

Figure 4 shows an example of our evaluation results, including the patient's fully exhaled image, fully inhaled image, the estimated image for one breathing phase, and the ground truth. We can see that the estimated lung image can well match the ground truth.

The proposed model based on the ridge regression is compared with the model based on interpolation[20]. As shown in Figure 5, the model errors using our proposed method are between $0.25 \mathrm{~mm}$ and $0.88 \mathrm{~mm}$ with an average of $0.51 \pm 0.25 \mathrm{~mm}$, while the interpolation methods' error are between $0.36 \mathrm{~mm}$ and $1.13 \mathrm{~mm}$ with an average of $0.62 \pm 0.31 \mathrm{~mm}$. The results showed that our proposed model outperforms interpolation-based model.
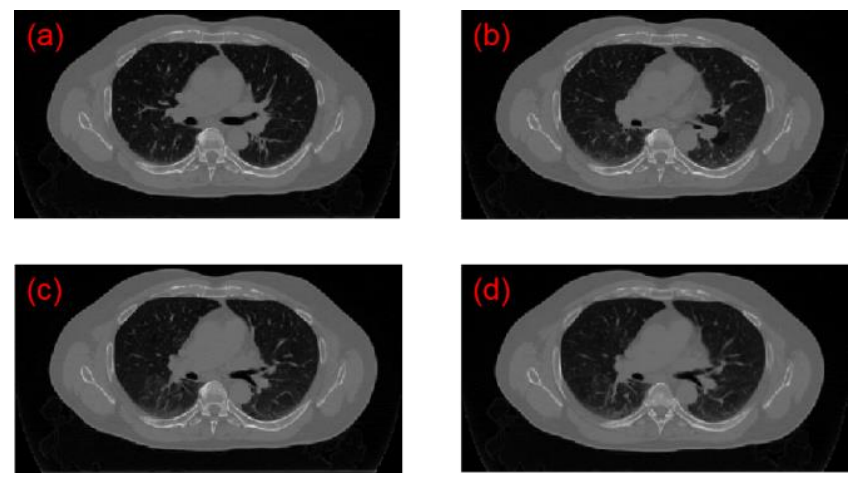

Figure 4: An example of the estimation result. The inhale

(a) and exhale (b) images, (c) current real image, (d) estimated image calculated by the respiratory model.

\section{Discussion}

In imaging guided diagnosis and intervention,respiratory motion is a key factor to affect the accuracy. Respiratory motion model is found a quantitative relationship between surrogate motion and lung internal motion and estimate the lung motion in real time. The limited dimensions of the surrogate signal may not be able to estimate accurate lung motion. High-dimensional chest surface motion can be acquired by segmented surface[18].

The correlation between the chest surface motion and the lung internal motion is a key factor to affect the accuracy of the proposed model. In this work, we investigated the level of correlation between the motion of different external regions and internal landmarks. These regions which have been shown higher correlation coefficient were chosen to establish respiratory motion model.

Most previous respiratory motion models require $4 \mathrm{D} \mathrm{CT}$ for each patient, leading radiation dose increase. Sarrut et al.[20] assumptions that each particle displacement is linear and proposed the interpolation-based model that obtained artificially $4 \mathrm{D}$ CT from exhale and inhale image. However, hysteresis problem is not considered in this model. Therefore, we calculated specific patients' 4D CT based on two static 3D CT with ridge regression algorithm, and there is no need to collect $4 \mathrm{D} C \mathrm{CT}$ images. This model is simultaneously considering global common features and individual specific characteristics of lung motion. Experiments demonstrate that the proposed model outperforms interpolation-based model and achieved a better balance between predictive accuracy and radiation doses. In percutaneous lung cancer intervention, this model could be used to navigate the interventional device.

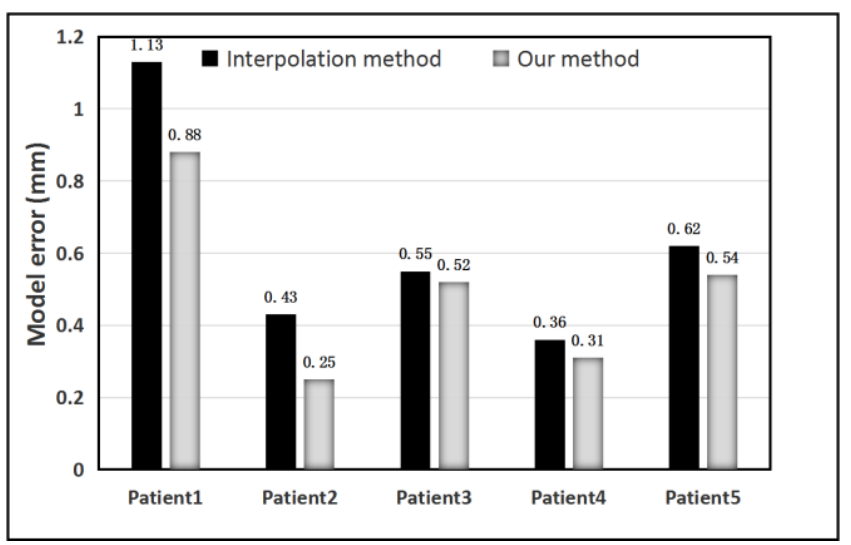

Figure 5: Average errors for respiratory motion model.

\section{Conclusions}

In this paper, we have developed a new patient-specific respiratory motion model. This model establishes the mathematical relationship between lung internal motion and external chest surface motion. Only two static 3D CT are needed for a specific patient to derive $4 \mathrm{D} \mathrm{CT}$. The estimated lung image from respiratory model and acquired real 4D CT can be matched well. In the future, the proposed model could be used in imageguided percutaneous lung biopsy and radiofrequency ablation.

\section{Acknowledgement}

This work was supported by the National Key R\&D Program of China (2017YFB1300204), Hefei Foreign Cooperation Project (ZR201801020002), the Natural Science Fund of Anhui Province (2008085MC69), Collaborative Innovation Program of Hefei Science Center (2020HSC-CIP001), CAS Anhui Province Key 
Laboratory of Medical Physics and Technology (LMPT201904) and Director's Fund of Hefei Cancer Hospital of CAS (YZJJ2019C14, YZJJ2019A04) to TW, ZZ, QC, LZ, GX, LY, HW, $\mathrm{HL})$.

\section{References}

[1] Ronald S Winokur, Bradley B Pua, Brian W Sullivan, David C Madoff. 2013 Percutaneous Lung Biopsy: Technique, Efficacy, and Complications. Semin Intervent Radiol 30, 121-127. https://doi.org/10.1055/s-0033-1342952.

[2] Stuart S C Burnett, Katharina E Sixel, Patrick C F Cheung, Jeremy D P Hoisak. 2008. A study of tumor motion management in the conformal radiotherapy of lung cancer. Radiotherapy and Oncology. 86(1): 77-85. https://doi.org/10.1016/j.radonc.2007.11.017.

[3] JR McClelland, DJ Hawkes, T Schaeffter, AP King. 2013. Respiratory motion models: a review. Med. Image Anal 17:19-42. https://doi.org/10.1016/j.media.2012.09.005.

[4] Jamie R McClelland, Marc Modat, Simon Arridge, Helen Grimes, Derek D’Souza, David Thomas, Dylan O' Connell, Daniel A Low, Evangelia Kaza, David J Collins, Martin O Leach, David J Hawkes. 2017. A generalized framework unifying image registration and respiratory motion models and incorporating image reconstruction, for partial image data or full images. Phys. Med. Biol., 62, 4273. https://doi.org/10.1088/1361 -6560/aa6070.

[5] Hui Lin, Chengyu Shi, Brian Wang, Maria F Chan, Xiaoli Tang, Wei Ji. 2019. Towards real-time respiratory motion prediction based on long short-term memory neural networks. Phys. Med. Biol., 64, 085010 https://doi.org/10.1088/1361-6560/ab13fa.

[6] Shahabedin Nabavi, Monireh Abdoos, Mohsen Ebrahimi Moghaddam, Mohammad Mohammadi. 2020. Respiratory Motion Prediction Using Deep Convolutional Long Short-Term Memory Network. Journal of $\begin{array}{llll}\text { Medical Signals and Sensors } & \text { 10(2): }\end{array}$ https://doi.org/10.4103/jmss.JMSS_38_19.

[7] Qi Fan, Xiaoyang Yu, Yanqiao Zhao, Shuang Yu. 2020. A Respiratory Motion Prediction Method Based on Improved Relevance Vector Machine. Mobile Networks and Applications 25:2270-2279. https://doi.org/10.1007/s11036-02001610-7.

[8] Tiancheng He, Zhong Xue, Nam Yu, Paige L Nitsch, Bin S Teh, Stephen T Wong. 2014. Estimating Dynamic Lung Images from High-Dimension Chest Surface Motion Using 4D Statistical Model. In:Proceedings Medical Image Computing and Computer-Assisted Interventions MICCAI, Part II, LNCS 8674 pp. 138-145. https://doi.org/10.1007/978-3-319-10470-6_18.

[9] Qinghui Zhang, Alex Pevsner, Agung Hertanto, Yu-Chi Hu, Kenneth E Rosenzweig, C Clifton Ling, Gig S Mageras. 2007. A patient-specific respiratory model of anatomical motion for radiation treatment planning. Med. Phys. 34, 4772-4781. https://doi.org/10.1118/1.2804576.

[10] J R McClelland, S Hughes, M Modat, A Qureshi, S Ahmad, D B Landau, S Ourselin, D J Hawkes. 2011. Inter-fraction variations in respiratory motion models. Phys. Med. Biol., 56 (2011), 251-272. https://doi.org/10.1088/00319155/56/1/015.

[11] Hadi Fayad, Tinsu Pan, Olivier Pradier, Dimitris Visvikis. 2012. Patient specific respiratory motion modeling using a 3D patient's external surface. Med. Phys., 39 (6), 3386-3395. https://doi.org/10.1118/1.4718578.

[12] Joo-Hyun Chung, Minsoo Chun, Jung-in Kim, Jong Min Park, Kyung Hwan Shin. 2020. Three-dimensional versus four-dimensional dose calculation for breast intensity-modulated radiation therapy. BJR 108:e321-e322. https://doi.org/10.1259/bjr.20200047.

[13] Nakamura M, Ishihara Y, Matsuo Y, et al (2018) Quantification of the kV X-ray imaging dose during real-time tumor tracking and from three- and fourdimensional cone-beam computed tomography in lung cancer patients using a Monte Carlo simulation. J Radiat Res 59:173e181. https://doi.org/10.1093/jrr/rrx098.

[14] A. Sam Beddar,Kristofer Kainz,Tina Marie Briere, Yoshikazu Tsunashima, Tinsu Pan, Karl Prado, Radhe Mohan, Michael Gillin, Sunil Krishnan. 2007. Correlation between internal fiducial tumor motion and external marker motion for liver tumors imaged with 4D-CT. Int. J.Radiat. Oncol., Biol., Phys.67(2), 630-638 (2007). https://doi.org/10.1016/j.ijrobp.2006.10.007.

[15] E Kanoulas, J A Aslam, G C Sharp, R I Berbeco, S Nishioka, H Shirato, S B Jiang. 2007. Derivation of the tumor position from external respiratory surrogates with periodical updating of the internal/external correlation. Phys. Med. Biol.52, 5443-5456 (2007). https://doi.org/10.1088/0031-9155/52/17/023.

[16] Tiancheng He, Zhong Xue, Weixin Xie , Stephen T Wong. 2010. Online 4-D CT Estimation for Patient-Specific Respiratory Motion Based on Real-Time Breathing Signals. MICCAI Part III 6363:392-399. https://doi.org/10.1007/9783-642-15711-0_49.

[17] MJ Ledesma-Carbayo, J Kybic, M Desco, A Santos, M Suhling, P Hunziker, M Unser. 2005. Spatio-temporal nonrigid registration for ultrasound cardiac motion estimation. IEEE Trans. Med. Imaging,24(9),1113-1126. https://doi.org/10.1109/TMI.2005.852050

[18] Xiangrong Zhou, Takeshi Hara,Hiroshi Fujita, Ryujiro Yokoyama, Takuji Kiryu, Hiroaki Hoshi. 2004. Automated segmentations of skin, soft-tissue and skeleton from torso CT images. Proc. SPIE5370, 1634-1639. https://doi.org/10.1117/12.534843.

[19]T Wentz, H Fayad, J Bert, O Pradier, J F Clement, S Vourch, N Boussion, D Visvikisl. 2012. Accuracy of dynamic patient surface monitoring using a timeof-flight camera and B-spline modeling for respiratory motion characterization. Phys Med Biol. 57:4175-4193. https://doi.org/10.1088/0031 9155/57/13/4175.

[20] David Sarrut, Vlad Boldea, Serge Miguet, Chantal Ginestet. 2006. Simulation of four-dimensional CT images from deformable registration between inhale and exhale breath-hold CT scans.Med Phys. 33:605-617. https://doi.org/10.1118/1.2161409. 